\title{
Die Verwendung von Zahlsubstantiven zur Bezeichnung von Monatstagen in den griechischen Papyri
}

\author{
Dieter Hagedorn (Köln)
}

\begin{abstract}
In this article the author tries to answer the question, whether a general rule of the ancient Greek language, i.e. to designate the fourth, the twentieth, and the thirtieth days of a

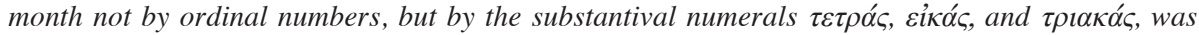
also observed in the Greek papyri and ostraca from Egypt.
\end{abstract}

Keywords: Greek language, substantival numerals, days of a month

Eine Eigentümlichkeit der griechischen Sprache bei der Verwendung von Zahlen bestand darin, daß zur Bezeichnung der Tage eines Monats nicht in jedem Fall

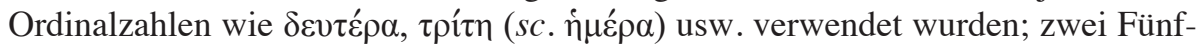
tel der Tage benannte man vielmehr auf andere Weise, und zwar - abgesehen vom Monatsersten, der gewöhnlich nicht $\pi \rho \omega ́ \tau \eta$, sondern $v \varepsilon o \mu \eta v i ́ \alpha$ hie $\beta^{1}-$ mithilfe der Zahlsubstantive $\tau \varepsilon \tau \rho \alpha ́ c$ (für den 4. Tag), sî́ác (für den 20. sowie - in Verbindung

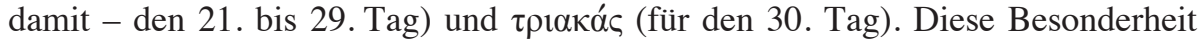
läßt sich in der Literatur ebenso wie in der Alltagssprache beobachten und ist daher natürlich auch in den einschlägigen Darstellungen und Grammatiken behandelt worden. ${ }^{2}$ Allerdings finden sich in den Editionen vereinzelte Zeugnisse, die zu belegen scheinen, daß auch Abweichungen vorkamen, man also nicht von einer strengen Regel sprechen kann. Mir scheint es daher berechtigt, einmal der Frage nachzugehen, wie konsequent man an der genannten Eigentümlichkeit, die man auch als „Inkonsequenz“ empfinden könnte, speziell in Ägypten festgehalten hat,

\footnotetext{
${ }^{1}$ Selbst der erste der fünf (oder sechs) Zusatztage am Jahresende, der غ̇ं $\alpha \gamma o ́ \mu \varepsilon v \alpha$, hieß $v \varepsilon o \mu \eta-$ vía; vgl. P.Köln II 100,1 und P.Oxy. LII 3691,4. Bei flüchtiger Suche in den Papyri und Ostraka

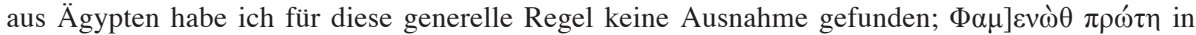

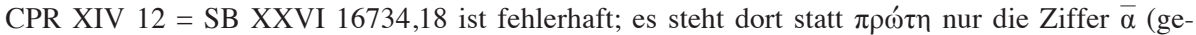
sprochen und ausgeschrieben wurde das eben veounvía); vgl. BL X 54 und N. Gonis in ZPE 159

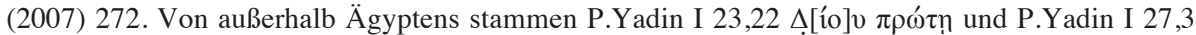

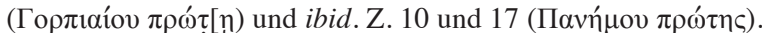

2 Ich verweise nur auf K. Meisterhans, Grammatik der attischen Inschriften (1900), S. 163f.; E. Mayser, Grammatik der griechischen Papyri aus der Ptolemäerzeit, Bd. I 2 (1938), S. 78 unter B 4; F. Gignac, A Grammar of the Greek Papyri of the Roman and Byzantine Periods, Bd. II (1981), S. 205 unter B 4. Ihrer umfassenden Zielsetzung entsprechend sind die Darstellungen in dieser speziellen Frage allerdings wenig detailliert und bisweilen sogar unzutreffend (s. die Richtigstellung eines bei Gignac falsch dargestellten Details unten, Fußn. 19).
} 
d.h. weitab vom griechischen Mutterland und in einer Umgebung, in der die autochthone Bevölkerung, zumal die der Chora, Griechisch im besten Fall als Zweitsprache beherrschte; ein laxerer Umgang mit den Feinheiten der Sprache, gewissermaßen eine Normalisierung, wäre hier verständlich. Ägypten ist zudem als Objekt einer derartigen Untersuchung besonders geeignet, einmal, weil wir von dort in den Papyri und Ostraka ein ganz ungewöhnlich umfangreiches Corpus von schriftlichen Äußerungen aller Bevölkerungsschichten, sowohl der gebildeten als auch der weniger gebildeten oder gar deutlich ungebildeten, besitzen, zum anderen aber, weil dieses große Textcorpus durch die elektronische Textdatenbank „Duke Databank of Documentary Papyri“ nahezu vollständig erschlossen und entsprechend leicht recherchierbar ist. ${ }^{3}$ Meine folgende Musterung der Termini

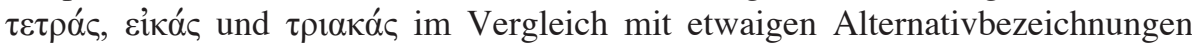

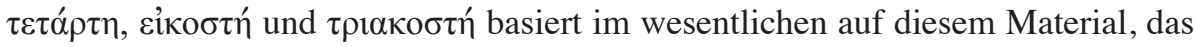
ich aber zusätzlich durch noch nicht in der DDbDP erfaßte Papyri und Ostraka erweitert habe. ${ }^{4}$

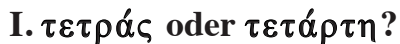

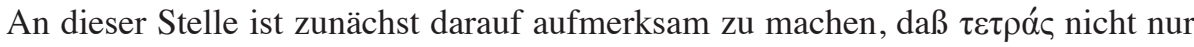
für den 4. Tag des Monats, sondern - in Verbindung mit cíkás - auch für den 24. verwendet wurde, so daß es eine teilweise Überschneidung mit dem folgenden Abschnitt II. gibt. Ich werde hier das generelle Auftreten des Wortes behandeln, dagegen die Besonderheiten der Verbindung mit cikás weiter unten.

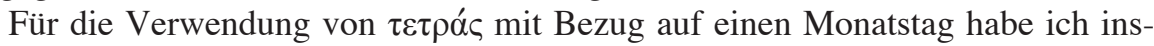
gesamt 41 sichere Belege finden können; davon bezeichnen 25 Belege den 4. Tag 5 und 15 Belege den 24. Tag, ${ }^{6}$ während sich in einem Fall ${ }^{7}$ wegen des fragmentarischen Zustandes des Papyrus nicht entscheiden läßt, welcher der beiden Tage ge-

3 Die DDbDP wird jetzt unter dem Dach des „Papyrological Navigator“ fortgeführt, der im Internet unter der Adresse <http://papyri.info/> erreichbar ist.

4 U.a. durch Einbeziehung der in den „Wörterlisten“ (http://www.papy.uni-hd.de/WL/ WL.html) gesammelten Belege. Nur gelegentlich habe ich auch aus Ägypten stammende epigraphische Quellen einbezogen; ich glaube nicht, daß sich das Bild bei stärkerer Benutzung der Inschriften verändert hätte.

5 In chronologischer Abfolge: P.Hib. I 65,10f. (ca. 265 v.Chr.); BGU VI 1284,4 (ca. 100 v.Chr.); O.Wilck. 9,4 (14-36); P.Merton I 10,2 (21); SB I 5252,14 (65); BGU I 251,1 (81); P.Med. I 59,3 (98-114); P.Lips. I 106,25 (99); P.Soterichos 25,18 (109); P.Wisc. II 80,188f. (114); P.Lips. II 140,7 (133); P.Oxy. LII 3690,4 (139); P.Mil. Vogl. III 145,27f. (142); P.Ross. Georg. II 26,6a (160); P.Ryl. II 197a,7 (162); P.Tebt. II 318,5 (166); P.Customs 305,3f. (185?); P.Mil. Vogl. III 202,15 (II); P.Strasb. I 12,10 (II?); BGU VII 1593,6.13 (spätes II-III); P.Lond. III 964 (S. 211),23 (spätes II-III); SB XXVI 16740,6 (210); O.Mich. I 316,2 (III); P.Oxy. VIII 1163,2 (V).

${ }^{6}$ P.Eleph. DAIK 3,12-14 (III v.Chr.); P.Dion. 20,9 (105 v.Chr.); P.Tebt. I 105,9f. (103 v.Chr.); P.Dime III 5,1 (11); P.Mich. V 334,3 (52); P.Soterichos 11,15f. (77); P.Oxy. XXXVI 2759,14f. (116); P.Ryl. II 157,24 (135); PSI VIII 961 B,54 (178); P.Aberd. 40b,6-8 (190); P.Alex. Giss. 11,4-6 (Ende II - Anfang III); P.Customs 332,5 (208); P.Customs 343,4f. (210); P.Customs 344,5f. (210); P.Lond. VI 1914,6 (335?).

${ }^{7}$ P.Strasb. VIII 708,1 Tô]ßı $\varepsilon \varepsilon \tau \rho \alpha ́ \delta[1$. 
meint ist. Sie treten mit ersten Beispielen in der frühen Ptolemäerzeit auf und reichen in dichter Abfolge bis an den Anfang des 3. Jh. n.Chr.; die letzten gehören dem 4. Jh. (um das Jahr 335 n.Chr.) und vielleicht sogar (nicht näher datierbar) dem 5. Jh. n.Chr. an. ${ }^{8}$

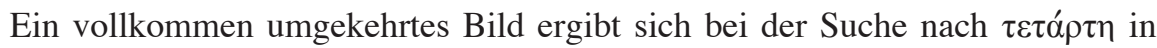
Verbindung mit einem Monatstag: Die unbezweifelbaren 18 Zeugnisse für den 4. Tag, die ich in ägyptischen Texten habe finden können, ${ }^{9}$ beginnen erst im 5. Jh. und erstrecken sich bis in das 7. Jh.; ${ }^{10}$ zu ihnen treten noch zwei Zeugnisse für den 24. Tag (eines davon weitgehend ergänzt) ${ }^{11}$ sowie ein Beleg, der auf beide Weisen interpretiert werden könnte, ${ }^{12}$ hinzu, die alle ebenfalls der Spätzeit angehören.

Dieses klare Ergebnis erfordert eine kritische Betrachtung von acht unsicher gelesenen bzw. ergänzten Beispielen, die sich nicht in das bisher gewonnene Bild einfügen ließen, wären sie zutreffend. Meines Erachtens sind sie allesamt zu korrigieren; es sind die folgenden:

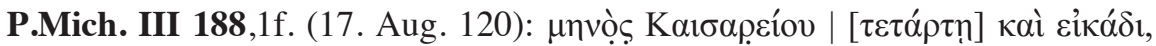

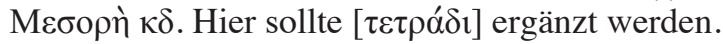

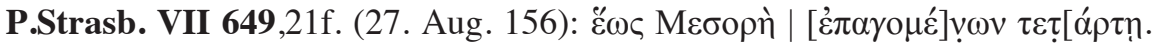
Hier sollte $\tau \varepsilon \tau$ [ $\rho$ ódoc ergänzt werden.

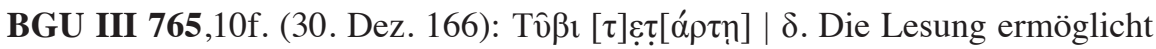

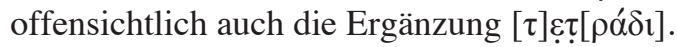

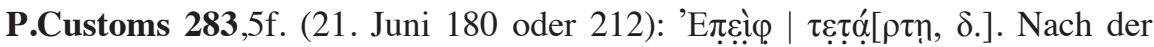

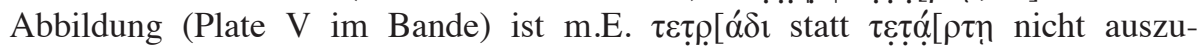
schließen.

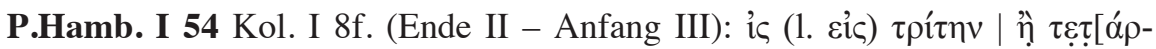

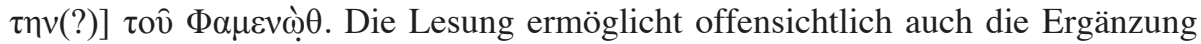

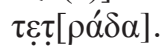

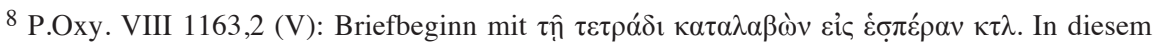
letzten Beispiel, welches die Herausgber mit „I arrived on the 4th“ übersetzen, könnte man sich jedoch fragen, ob mit $\tau \varepsilon \tau \rho \alpha ́ \varsigma$ nicht schon der Mittwoch gemeint ist, s. unten, Fußn. 15.

9 Wegen der außerägyptischen Herkunft sind folgende Vorkommen hier außer Betracht gelas-

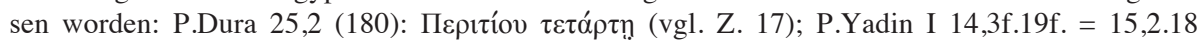

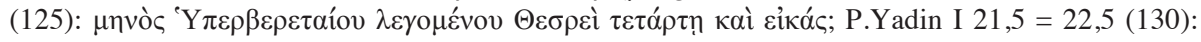

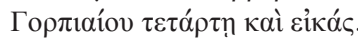

${ }^{10}$ Hier die vollständige, chronologisch geordnete Liste: P.Oxy. XVI 1982,18 (497); P.Heid. IV 333,12 (V); P.Herm. 27,3 (V); P.Lond. III 1313 (S. 256),2 (507); P.Cair. Masp. I 67107,2 (525 oder 540); P.Oxy. XXXVI 2779,19 (530); BGU XIX 2809,2 (533); P.Oxy. XVI 1983,19 (535); P.Cair. Masp II 67128,5 (547); P.Cair. Masp. III 67302,2 (555); P.Michael. 42B,2 (566); P.Cair. Masp. II 67163,4 (569); SB VI 9293,2 (573); BGU XII 2206,5 (591-602); P.Ross. Georg. III 45,5f. (VI); P.Lond. V 1875,3 (615/6 oder 630/1); SB I 4483,2 (vgl. APF 53 [2007] 154-167; 621); P.Apoll. 9,1 (660/1 oder 675/6).

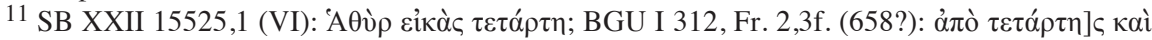

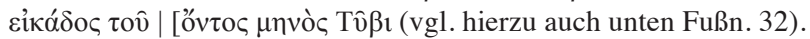

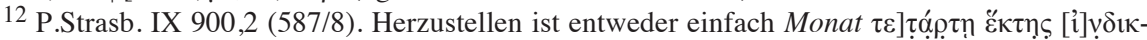

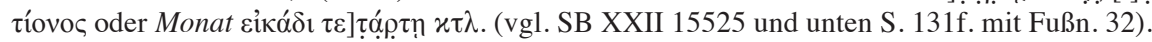




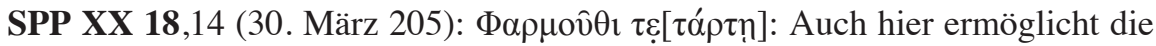
Lesung die Ergänzung $\tau € ̣[\tau \rho \alpha ́ \delta ı]$.

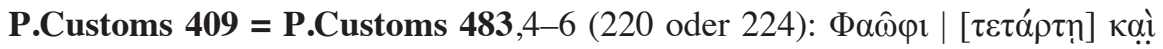

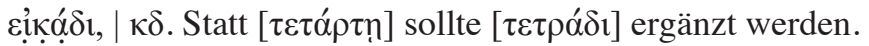

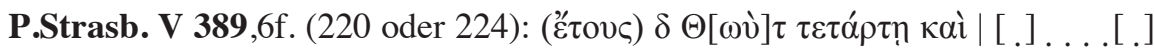
1 $\delta$. Im Kommentar zu Z. 7 schreibt J. Schwartz: „Au début, les traces ne cor-

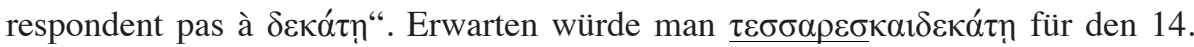

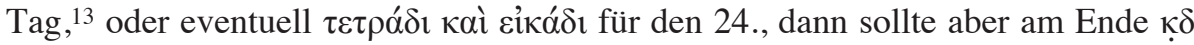
stehen. Paul Heilporn hat dankenswerterweise auf meine Bitte hin das Original überprüft und schreibt (E-Mail vom 7. Febr. 2013): „... le texte est particulière-

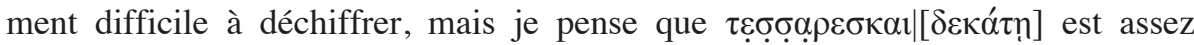

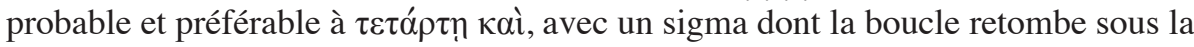
ligne d'écriture, ce qui a pu le faire passer pour un $\eta$. Au début de la 1.7, les traces d'encre sont tellement légères que je mettrais des crochets; devant le chiffre $\delta$, il ne reste que la pointe inférieure du iota de la dizaine, sans doute suffisant pour exclure $\kappa . " “$

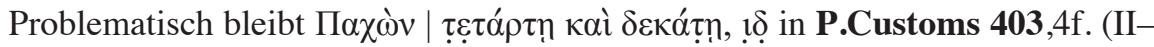
III), wo ebenfalls - dem zu dieser Zeit üblichen Sprachgebrauch entsprechend -

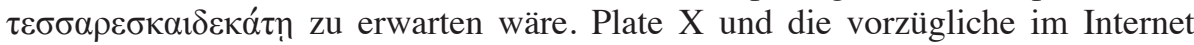
verfügbare Abbildung (s. dazu das HGV) lassen zwar erkennen, daß anstelle von

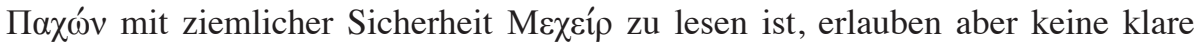

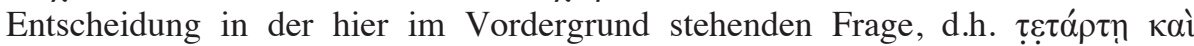

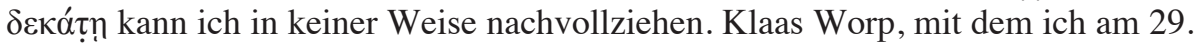
Aug. 2012 über den Text korrespondieren konnte, wofür ich ihm danke, bestätigte

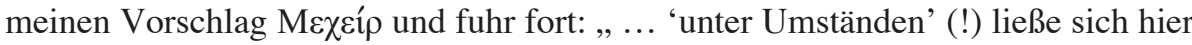
daran denken, ... mit ausgeschriebenem $\pi \varepsilon v \tau \varepsilon \kappa \alpha \iota \delta \varepsilon \kappa \alpha ́ \tau\rceil ̣, ~ \imath \varepsilon$ (mit Zahlmarkierung) zu rechnen." Dieser vorsichtigen Einschätzung stimme ich zu, habe aber im Detail weiterhin größte Schwierigkeiten.

Schließlich ist ein weiterer Text zu korrigieren, in dem das Auftreten von

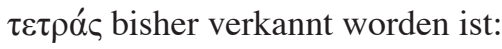

13 Zur generellen Bildeweise der Ordinalia für den 13.-19. Tag in nachklassischer Zeit s. Gignac (wie Fußn. 2), S. 202-204. Abweichungen davon kommen wohl vor, sind aber grundsätzlich suspekt und sollten einmal systematisch überprüft werden. Ein Beispiel: Die Suche nach

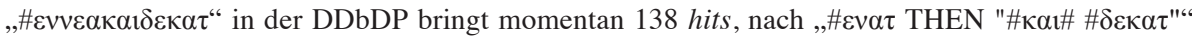
within 15 chars“ dagegen nur 8 hits. Von diesen 8 Stellen sind zunächst 4, an denen nicht „19.“, sondern „9. und 10.“ gemeint ist, auszuscheiden (P.Batav. 22,4f.; P.Oxy. IV 731,4f.; P.Athen. 51v,3 [das Verso ist in der Neudition SB XVI 12238 nicht mit abgedruckt worden]; SPP III 61,4f.). Zu SB XII 11053,1 s. jetzt die Diskussion in P.Sorb. III, S. 30f., wo gegen die Ergänzung ह̌ं

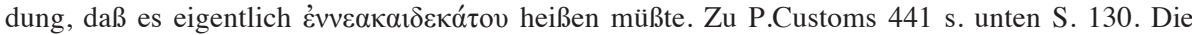
beiden restlichen Fälle (O.Mich. I 278,5f. und SB VI 9586,4) erscheinen mir an den Abbildungen äußerst fragwürdig. 


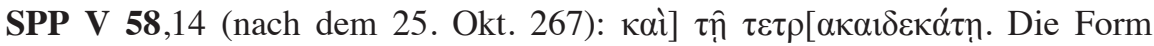
$\tau \varepsilon \tau \rho \alpha \kappa \alpha \iota \delta \varepsilon ́ \kappa \alpha \tau о \varsigma$ existiert im regulären Griechischen nicht; ${ }^{14}$ es sollte, falls der 14.

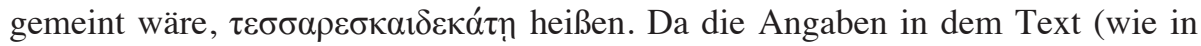
den Parallelen; vgl. u.a. SPP V 59,10ff.) innerhalb eines Monats chronologisch voranschreiten und in Z. 15f. vom 27. Tag die Rede ist, käme hier gut der 24. in

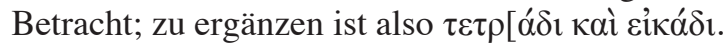

Fazit: Der Befund läßt keinen anderen Schluß zu, als daß sich zwischen ca. 335 und 497 n.Chr. in Ägypten ein Wandel vollzogen hat: ${ }^{15}$ Während man hier bis dahin zur Bezeichnung von Monatstagen ausnahmslos, soweit wir sehen, ${ }^{16}$ die traditionelle Bezeichnung $\tau \varepsilon \tau \rho \alpha ́ c$ verwendet hat, ist diese im Laufe der 2. Hälfte des

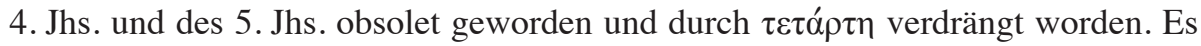
steht zu vermuten, daß der Wechsel nicht abrupt erfolgte, sondern es eine Über-

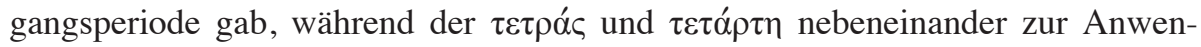
dung kommen konnten.

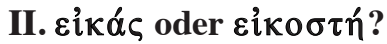

Für die Verwendung von દỉkác zur Bezeichnung eines Monatstages lassen sich gegenwärtig 385 Beispiele in den ägyptischen Papyri und Ostraka finden; davon betreffen 89 den 20. Tag selbst, die restlichen verteilen sich auf die folgenden Tage (21.-29.) oder sind wegen der Lücken im Text nicht eindeutig einem Tag zuzuordnen. Sie stammen aus allen Epochen, in denen Griechisch in Ägypten in den Alltagstexten verwendet wurde, d.h. von der Ptolemäerzeit bis in die Spätantike, genauer gesagt vom 3. Jh. v.Chr. bis in das 7./8. Jh. n.Chr. ${ }^{17}$

14 Das zweite Vorkommen von $\tau \varepsilon \tau \rho \alpha \kappa \alpha 1 \delta \varepsilon ́ \kappa \alpha \tau o \varsigma$, das die DDbDP verzeichnet, beruht auf einem unerklärlichen Fehler in der Edition von O.Claud. III 547; das Ostrakon hat in Z. 6f. die geringfügige orthographische Abweichung $\tau \varepsilon \sigma \alpha \rho \varepsilon \sigma|\kappa \alpha 1 \delta \varepsilon \kappa \alpha ́ \tau o v ~ s t a t t ~ \tau \varepsilon \sigma \sigma \alpha \rho \varepsilon \sigma| \kappa \alpha 1 \delta \varepsilon \kappa \alpha ́ \tau o v$, aber

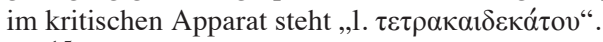

15 Es dürfte schwerfallen, Gründe für diese Veränderung zu finden. Denkbar wäre immerhin, daß sie mit der Tatsache im Zusammenhang zu sehen ist, daß um etwa dieselbe Zeit das Wort $\tau \varepsilon \tau \rho \alpha ́ c ̧$ zur Bezeichnung des 4. Wochentages, also des Mittwochs, üblich wurde; vgl. z.B. Lampe, Patristic Greek Lexicon, s.v. $\tau \varepsilon \tau \rho \alpha ́ c \varsigma$ 4; P.Mon. Epiph. 618 = P.Rain. Unterricht Copt. 252,9.18. Vielleicht wollte man eine Verwechselung vermeiden. Allerdings hatte man offenbar keine Beden-

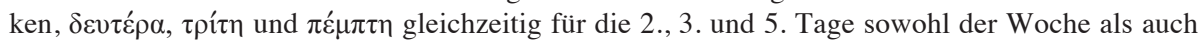
des Monats zu verwenden. S. auch oben Fußn. 8.

${ }^{16} \mathrm{Zu}$ außerägyptischen Ausnahmen s. oben Fußn. 9.

17 Wegen der großen Zahl von Zeugnissen beschränke ich mich auf die Nennung der 20 frühesten und 20 spätesten Beispiele: P.Eleph. 5,17f. (281 v.Chr.); SB XII 11053,2 (277 oder 276 v.Chr.); P.Cair. Zen. II 59150,7f. (256 v.Chr.); SB XIV 11308,11f. (251 v.Chr.); PSI IV 379,9f. (248 v.Chr.); P.Cair. Zen. III 59462,2 (246 v.Chr.); P.Tebt. III.1 815 Fr. 3r,2 (222 v.Chr.); BGU VI 1274,4 (218/7 v.Chr.); P.Eleph. DAIK 3,13f. (III v.Chr.); P.Zen. Pestm. 35,24 (III v.Chr.); P.Cair. Zen. III 59521,3 (Mitte III v.Chr.); P.Tebt. III.1 816,43 (192 v.Chr.); P.Tebt. III.1 817,7f. (182 v.Chr.); P.Amh. II 42,28f. (179 v.Chr.?); BGU VI 1272,5 (173 v.Chr.); P.Tebt. III.1 819,3f.22f. (171 v.Chr.); SB XIV 11966,7 (170-116 v.Chr.); P.Hamb. I 57,11 (160 v.Chr.); SB V 7632,4.27f. (159 v.Chr.); SB XXVI 16698,7 (152 v.Chr.) ... SPP XX 219,19 (604); BGU XVII 2694,5 (608); CPR X 1,9 (609; zur Lesung vgl. BL IX 71); P.Oxy. LXX 4800,14 (611?); P.Oxy. LXXII 4930,18 (614); P.Strasb. V 328,4 (618; vgl. unten S. 133); SB XVIII 13173,5.52.101.123 (629 oder 644); 
Die Art und Weise, wie die Tage vom 21. bis zum 29. ausgedrückt werden, verdient eine genauere Betrachtung.

Grundsätzlich erfolgt die Benennung durch die Verbindung einer Ordinalzahl mit sikác, aber es ist anzumerken, daß der 21. und - wie bereits oben dargelegt der 24. eine abweichende Behandlung erfahren: Im Falle des 21. wird statt der Ordinalzahl die Kardinalzahl ( $\left.\mu i^{\prime} \alpha\right)$ verwendet, ${ }^{18}$ im Falle des 24 . tritt das Zahlsub-

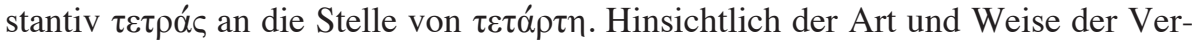
bindung sind drei Verfahren (A, B, C) zu unterscheiden, die zeitlich aufeinanderfolgen, sich teilweise aber auch überlappen. ${ }^{19}$

A. In der frühesten Zeit, d.h. im 3. Jh. und am Beginn des 2. Jh. v.Chr., finden wir in Ägypten nur einige wenige Beispiele für die Angabe mit einer Formel, die auch aus Inschriften ${ }^{20}$ und außerägyptischen Papyri (auch späterer Zeit) ${ }^{21}$ bekannt

SB XVIII 13962,3 (630 oder 645); SB XVI 12492,6f. (638); P.Paramone 18,25 (641?); SB VI 8988,2 (647); SB Kopt I 242,3f. (649); BGU I 323,9f. (651); BGU I 312, Fr. 2,3 (657 oder 658); P.Berl. Zill. 8,4 (663); BGU I 310,12 (663?); SPP VIII 1187,2 (VII); SB I 4733,2 (VII); SPP III² ${ }^{2} 1$ 115,5.6 (VII-VIII); SPP VIII 1077,8 (VII-VIII).

${ }^{18}$ Diese Erscheinung steht im Einklang mit der generellen Regel im klassischen Griechisch,

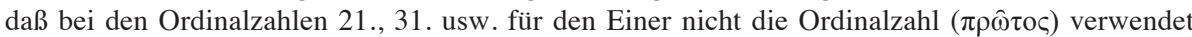

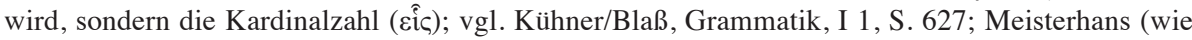
Fußn. 2), S. 164 unter 27; Gignac, Grammar II, S. 204 mit Fußn. 2. Die Regel wurde auch in Ägypten lange Zeit befolgt, wie Beispiele für die entsprechenden Regierungsjahre zeigen. So ver-

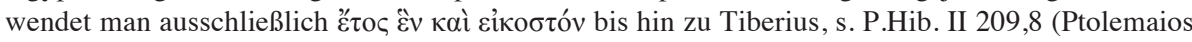
II.); P.Heid. VIII 414,17; SB XX 14991,12 (beide Ptol. V.); SB XX 14426,4f.9f. (Ptol. X.?); BGU XVI 2598,5; P.Amst. I 41,8.39; SB X 10573,5 (alle Augustus); BGU III 906,20; P.Kron. $48=$ P.Mich. V 260,20.39; P.Mich. V 263,19.20.36; 311,14f.; 312,16; P.Oslo II 33,2.10 (alle Tiberius).

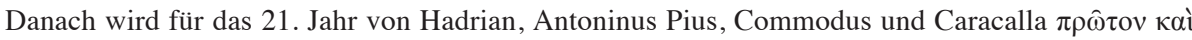

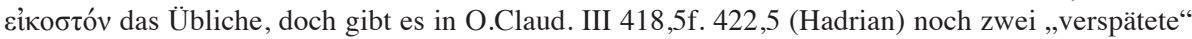

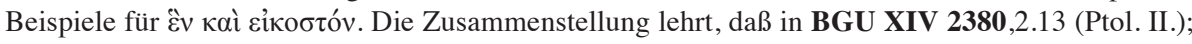
P.Tebt. I 176,2 (s. P.Yale I, S. 143) und P.Yale I 51,1 (beide Ptol. V) in den Lücken besser évóc

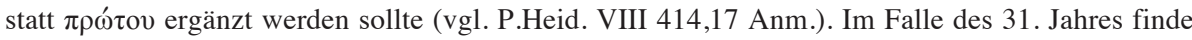

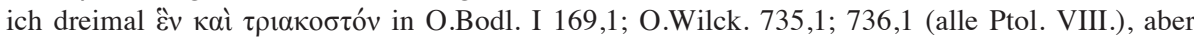

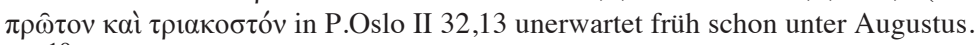

${ }^{19}$ Die Darstellung bei Gignac, Grammar II, S. 205 unter 4 b., ist bedauerlicherweise vollkommen unzutreffend. Nach seiner die Formel B beschreibenden Feststellung: „The unit precedes,

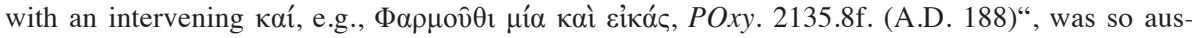
schließlich nur für die römische Zeit zutrifft, nicht mehr für die spätantike (s. unten unter C), fährt

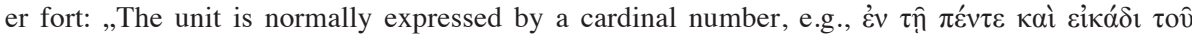

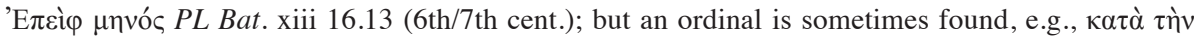

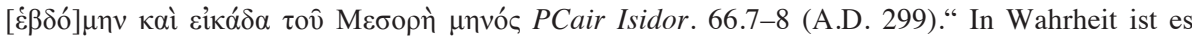
genau umgekehrt: Das Normale ist die Ordinalzahl, die Kardinalzahl ist eine Besonderheit beim 21. Tag. Zu dem Zitat von PL Bat. xiii 16 (= P.Select. 16) s. unten S. 130.

20 Vgl. dazu Meisterhans (wie Fußn. 2), S. 163 unter 25. Meisterhans führt allerdings keine

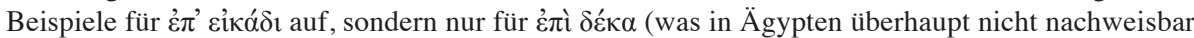
ist), aber die Inschriften-Datenbank des Packard Humanities Institute enthält auch etliche Beispiele für '̇ं $\pi^{\prime} \varepsilon i \kappa a ́ \delta 1$, darunter auch solche aus Ägypten, so das Kanopos-Dekret von 238 v.Chr. (OGIS I $56=$ St. Pfeiffer, Das Dekret von Kanopos, APF Beih. 18, München/Leipzig 2004).

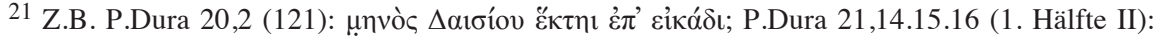

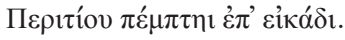




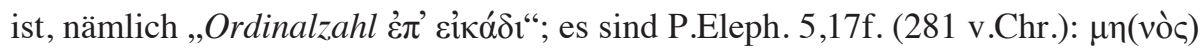

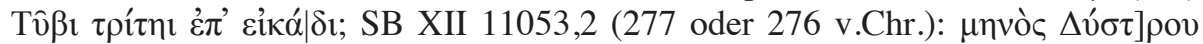

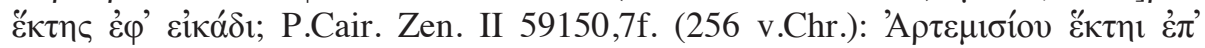

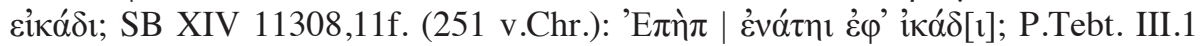

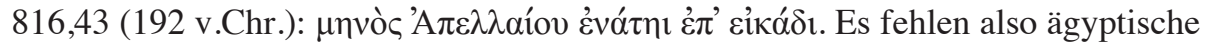
Zeugen für den 21. und den 24 . Tag, aber die Inschriften legen nahe, daß in diesen Fällen auch hier nicht die Ordinalzahl, sondern abweichend von der Grundregel

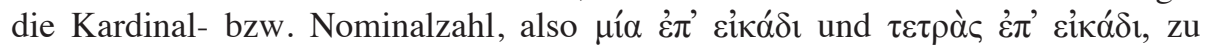
erwarten wären. ${ }^{22}$

B. Über einen Zeitraum von nahezu 800 Jahren ist die zweite Formel „Ordinalzahl кà દíkác“" bezeugt, in der also gegenüber Formel A die Konjunktion

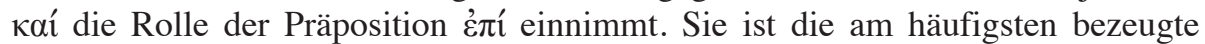
Formel überhaupt. Es haben sich für sie 258 indiskutable Beispiele finden lassen, die von 248 v.Chr. bis 657 oder 658 n.Chr. reichen;23 dies bedeutet. daß die Formeln A und B zeitweilig - d.h. für uns erkennbar von 248 bis 192 v.Chr. - nebeneinander zur Anwendung kommen konnten. Im Kanopos-Dekret OGIS I 56 (238 v.Chr.) erscheinen beide sogar innerhalb desselben Textes, s. Z. $34 \dot{\eta} \pi \dot{\varepsilon} \mu \pi \tau \eta \dot{\varepsilon} \pi$ '

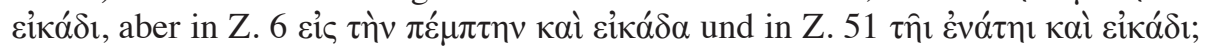
einen Unterschied in der Bedeutung oder der Funktion der beiden Formeln scheint es nicht zu geben.

Die 15 Belege für die abweichende Benennung des 24. durch Zahlsubstantiv

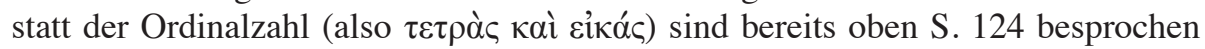

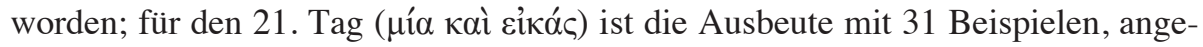
fangen mit P.Tebt. III.1 815, Fr. 3r,2 (5. Febr. 222 v.Chr.) bis zum spätesten exakt datierbaren SPP XX 54 Kol. I 11 (17. Juli 250), gut doppelt so groß. ${ }^{24}$

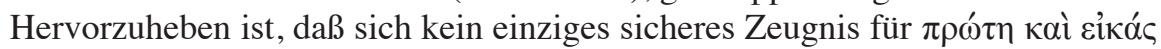

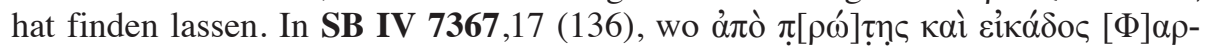

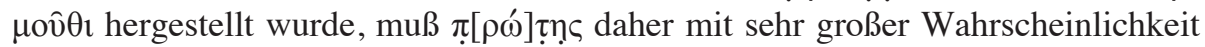
korrigiert werden, und zwar je nach Breite des verfügbaren Platzes am ehesten

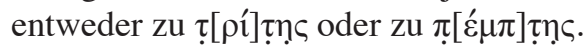

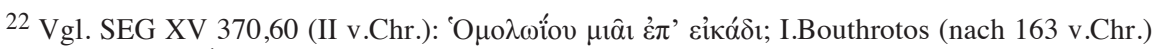

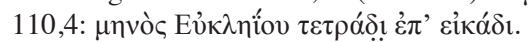

${ }^{23}$ Hier die zehn frühesten und zehn spätesten Exemplare: PSI IV 379,9f. (248 v.Chr.); P.Tebt. III.1 815 Fr. 3r,2 (222 v.Chr.); P.Eleph. DAIK 3,13f. (III v.Chr.); P.Zen. Pestm. 35,24 (III v.Chr.); P.Cair. Zen. III 59521,3 (Mitte III v.Chr.); P.Tebt. III.1 817,7f. (182 v.Chr.); P.Amh. II 42,28f. (179 v.Chr.?); BGU VI 1272,5 (173 v.Chr.); P.Tebt. III.1 819,3f.22f. (171 v.Chr.); P.Hamb. I 57,11 (160 v.Chr.) - - - P.Vindob. Sal. 9,2 (509); P.Cair. Masp. III 67301,4 (530); P.Oxy. LXX 4785,23 (530; s. Fußn. 29); P.Grenf. I 56,2 (537); CPR IX 32,13f. (551); PSI VII 836,13 (VI); SB I 4706,4 (VI-VII); P.Lond. III 871 (S. 269),22f. (603); SB XVIII 13962,3 (630 oder 645); BGU I 312 , Fr. 2,3 (657 oder 658).

${ }^{24}$ Ich verzichte auf einen Abdruck der vollständigen Liste und erwähne nur, daß P.Lond. III 1265 (d) (S. 37),4f. mit BL IX 142 zu lesen ist und daß an der fragmentarischen Stelle P.Amh. II

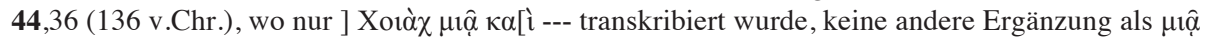

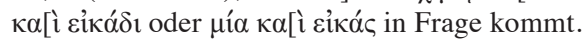


Wegen Gignacs Zitat und inkorrekter Interpretation von P.Select 16,13 (s. oben Fußn. 19) sei hier ausführlicher auf diesen Text eingegangen. In der Edition steht

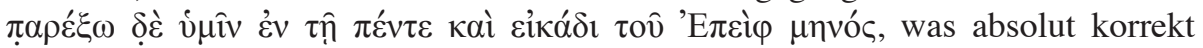
gelesen ist, wie man an der Abbildung im Bande (Abb. VII) kontrollieren kann,

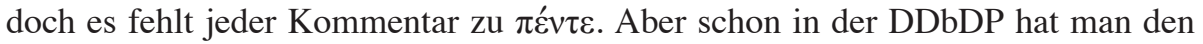

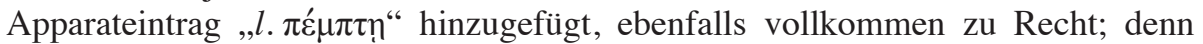

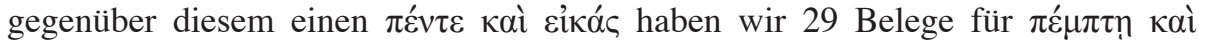

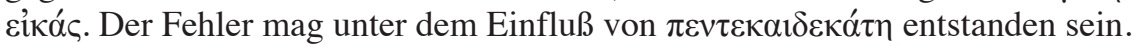

Zusätzliche Bemerkungen mit verwandter Thematik:

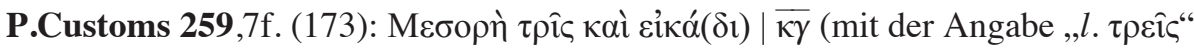
im Apparat und einem Verweis auf die unzutreffende Bemerkung bei Gignac).

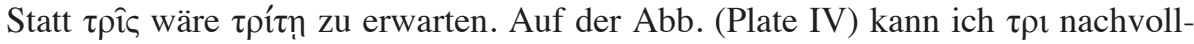
ziehen, kann die nachfolgende Schrift aber in keiner Weise deuten; ein Sigma danach halte ich für kaum vertretbar. Daß der 23. gemeint ist, mag zutreffen, wenngleich auch $\overline{\kappa \gamma}$ Punkte vertrüge; $1 \gamma$ ist als Lesung nicht möglich. - P.Customs

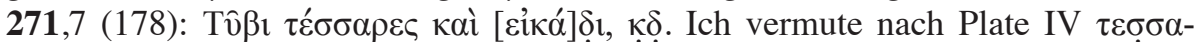

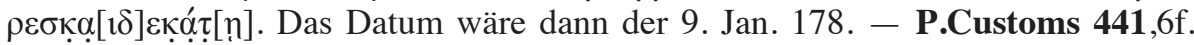

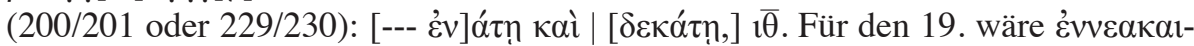

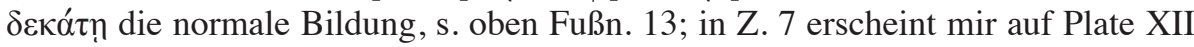

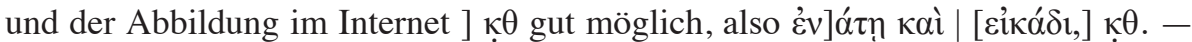

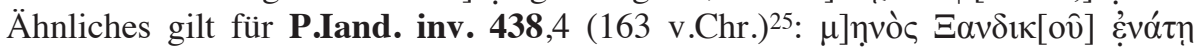
$\kappa \alpha[i$, wo hinsichtlich der Ergänzung im Kommentar gesagt wird: „Si potrebbe

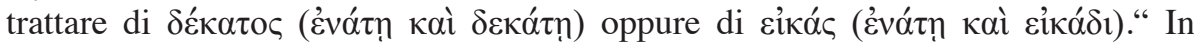
Wahrheit kommt grundsätzlich nur letzteres in Betracht. - Zu P.Oxy. LXX

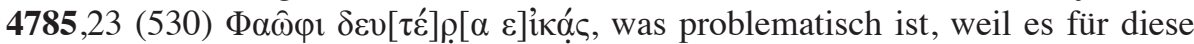
Verbindung ohne kaí keine Parallele gibt, vgl. unten Fußn. 29. - Ein weiteres

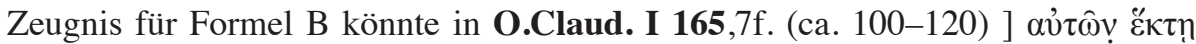

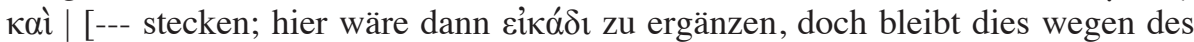
fragmentarischen Zusammenhangs unsicher. - In dem außerägyptischen SB X

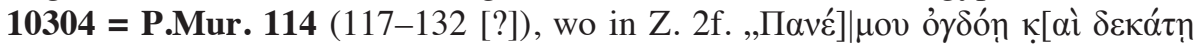

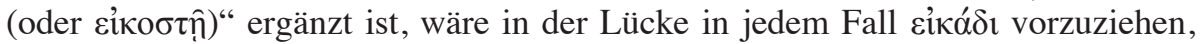

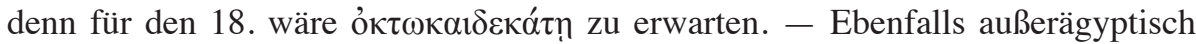

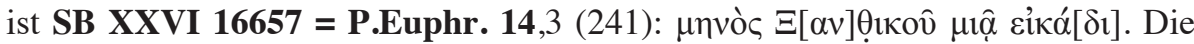
Lesung ist korrekt, aber wir sind in einem solchen Sonderfall vielleicht zu der

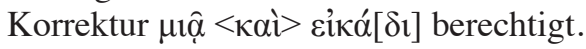

C. In der dritten Formel wird die Abfolge der Elemente vertauscht und auf deren Verbindung gänzlich verzichtet, sie lautet also einfach , ,ìkàc Ordinalzahl“. Für sie haben sich 28 sichere ägyptische Bezeugungen finden lassen, die alle dem 6. und dem 7. Jh. n.Chr. angehören; die früheste ${ }^{26}$ ist vermutlich P.Lond. III

${ }^{25}$ Soeben ediert von Marcin Kotyl, Due papiri tolemaici di Giessen, JJP 41 (2011) 67-78, hier 73-78.

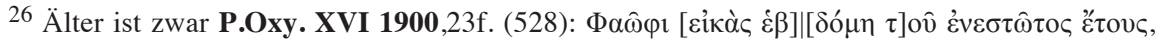

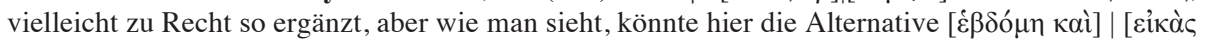


1040,2 aus dem Jahre 534, ${ }^{27}$ die späteste P.Berl. Zill. 8,4 (663). ${ }^{28}$ Wiederum können wir eine Überschneidung bei der Anwendung der Formeln B und C beobachten, ohne daß deutlich würde, warum man sich für die eine oder die andere Variante entschied. Ganz offenbar befand sich erstere auf dem Rückzug, wie ein Vergleich der Anzahl von Belegen beweist; denn den 28 Beispielen für $\mathrm{C}$ stehen aus demselben Zeitraum (d.h. 530 oder später) nur 5 oder 7 für B gegenüber, je nachdem ob man die beiden nur paläographisch datierten PSI VII 836,13 (VI) und SB I 4706,4 (VI-VII) mitrechnet oder nicht. ${ }^{29}$ Immerhin fällt auf, daß P.Grenf. I $56,2(537)^{30}$ das späteste Beispiel für die Verwendung von B in der Kopfdatierung einer Notariatsurkunde ist; in den anderen Fällen handelt es sich um weniger „offizielle“ Zeitbestimmungen. Eine Sonderbehandlung des 21. und des 24. Tages des Monats, die wir bei den Formeln A und B beobachten konnten, hat es bei der Formel $\mathrm{C}$ nicht gegeben; beide werden jetzt ebenfalls mit der Ordinalzahl

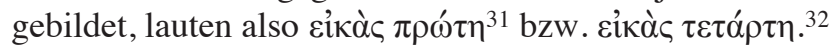

$\tau]$ ov kaum ausgeschlossen werden. Mit Sicherheit älter ist der außerägyptische Text P.Nessana 16,2 (512). - Vollkommen unverständlich und unerklärlich ist P.Tebt. II 422,16-18 (3. Jh.!):

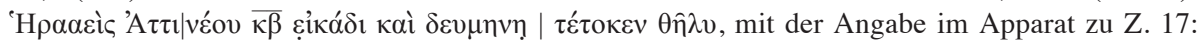

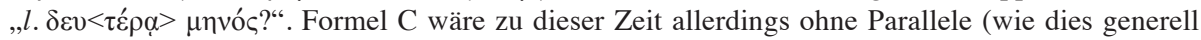
die vorangestellte Ziffer $\overline{\kappa \beta}$ ist).

27 Der Text wurde in ZPE 156 (2006) 226-232 neu ediert; zur Datierung vgl. ebenda, S. 228f.

28 Die vollständige Liste: P.Lond. III 1040 (Neuedtion ZPE 156 [2006] 226-232),2 (534); SB III 6704,11 (538); BGU XVII 2684,4 (555); P.Lond. III 1008 (S. 265),3 (561); P.Cair. Masp. II 67162,3.21 (568); P.Lond. V 1712,2 (569); P.Cair. Masp. II 67156,1 (570); P.Michael. 48,5 (572); P.Cair. Masp. I 67096,3 (573); P.Oxy. XVI 1894,10 (573); P.Münch. I 7,4 (583); P.Münch. I 13,3 (594); SB XX 14104,18 (VI); SB XXII 15525,1 (VI); P.Fouad I 86,1 (VI); SB XVIII 14054,4 (VIVII); P.Grenf. II 87,5 (602); CPR X 1,9 (609; zur Lesung vgl. BL IX 71); P.Oxy. LXX 4800,14 (611?); CPR IX 35.6 (613); P.Oxy. LXXII 4930,18 (614); BGU XII 2210,5 (617); SB XVIII 13173,5.52.101.123 (629 oder 644); SB XVI 12492,6f. (638); P.Paramone 18,25 (641?); SB VI 8988,2 (647); SB Kopt I 242,3f. (649); P.Berl. Zill. 8,4 (663). Mögliche weitere Beispiele sind P.Strasb. V 328,4 (618; vgl. unten S. 133) und P.Strasb. IX 900,2 (587/8; vgl. oben Fußn. 12).

29 Problematisch ist P.Oxy. LXX 4785,23 (530)

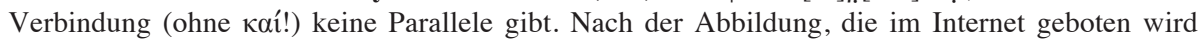

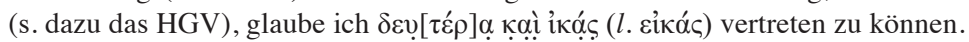

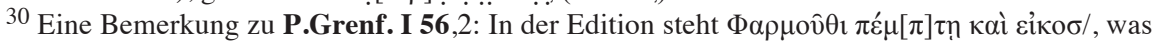

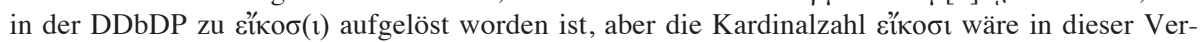
bindung ohne jede Parallele. Ich vermute, daß eine Form des Zahlsubstantivs hier steht, entweder

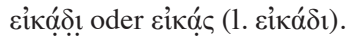

$3 \ddot{1}$ Vgl. P.Lond. V 1712,2 (569); BGU XII 2210,5 (617); SB XVIII 13173,5.52.101.123 (629

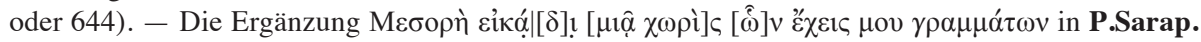

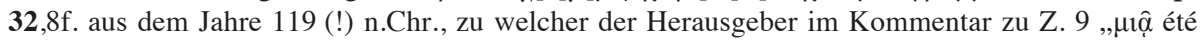
suggéré par l'étroitesse de la lacune“ anmerkt, ist natürlich vollkommen unmöglich; es kommt nur der 20. Mesore als Datierung in Frage, so daß die Lücke (wenn überhaupt; vielleicht gab es ein Spatium) anders gefüllt werden muß. Damit bleibt die Umrechnung „13. Aug.“ der Edition gegen

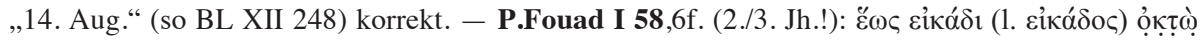

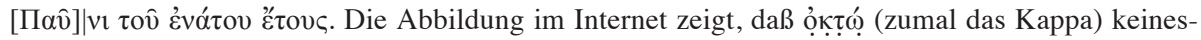

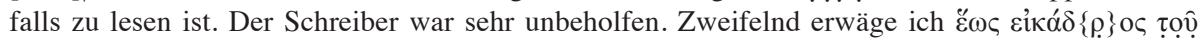
$\Pi[\alpha \hat{v}] \mid v \imath$. 


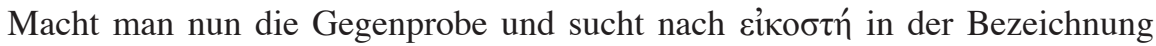
eines Monatstages, so findet man in den Editionen 11 mögliche Beispiele. Da ihnen die oben genannten 385 Beispiele für sikás in dieser Funktion gegenüberstehen, drängt sich die Frage auf, als wie zuverlässig die doch vergleichsweise wenigen Abweichungen anzusehen sind, zumal in einigen solcher Fälle die Lesungen schon in der Vergangenheit korrigiert worden sind. ${ }^{33}$ Acht von ihnen glaube ich ausscheiden zu können:

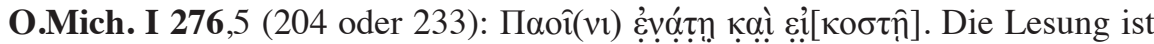
insgesamt sehr unsicher, wie die Abbildung im Internet zeigt, aber offenbar wäre

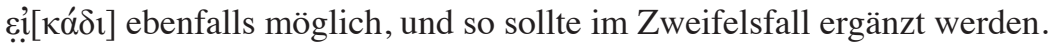

In der Torzollquittung P.Alex. Giss. 13 liest man in Z. 5-8 $\lambda \alpha \chi \alpha v o v ̣ \pi \varepsilon \dot{\varepsilon} \rho(\mathrm{ov}) \mid$

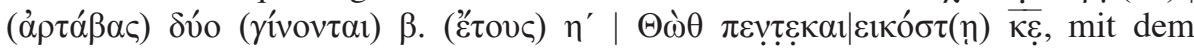

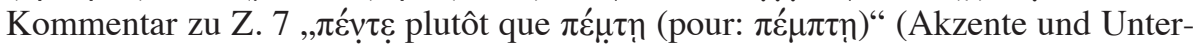
punktierung nach der Edition). Anhand eines digitalen Photos, für das ich mich

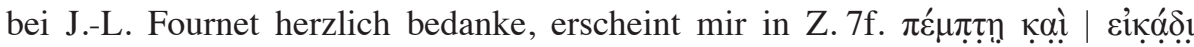
durchaus möglich, und dies ist daher vorzuziehen. Aber auch die vorangehenden Zeilen möchte ich anders lesen: In Z. 5 ziehe ich $\lambda \alpha \chi \alpha v o \sigma \sigma \pi \varepsilon \dot{\varepsilon} \rho \mu_{(0)}(v)$ anstelle von

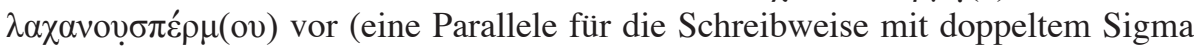
findet sich in O.Mich. VIII 464,15). In Z. 6 sehe ich nach dúo anstelle eines

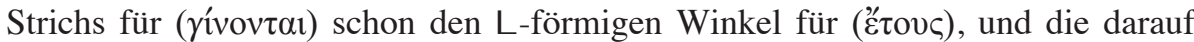
folgende Zahl könnte außer $\beta$ auch - und vielleicht eher $-\kappa$ sein, gefolgt nur von der Zahlmarkierung mit doppeltem Schrägstrich ( $)$. Meine Transkription von Z. 6

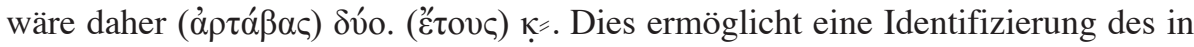

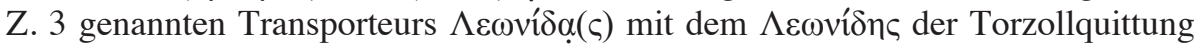
P.Grenf. II 50m, Z. 6, die ebenfalls in einem 20. Jahr abgefaßt worden ist und die man heute auf den 4. März 212 datiert (s. P.Customs 357); akzeptiert man diese Datierung, müßte P.Alex. Giss. 13 am 23. Sept. 211 geschrieben worden sein.

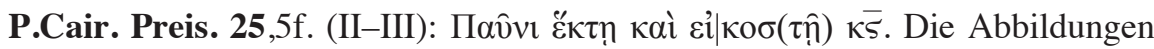
bei S. el Sawy und A. Bülow-Jacobsen, The Cairo-Preisigke Papyri. Plates, Cairo

32 SB XXII 15525,1 (VI), möglicherweise auch P.Strasb. IX 900,2 (587/8). - In BGU I 312, Fr. 2,3 (657 oder 658), dem spätesten bekannten Beispiel für die Formel B, ist wegen der Datie-

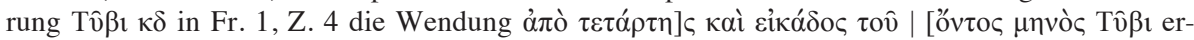
gänzt worden; grundsätzlich käme als Beginn der Laufzeit aber auch ein Folgetag in Frage; aus diesem Grunde mag ich weder den Text als einen Beleg für $\tau \varepsilon \tau$ ó $\rho \tau \eta$ werten, was allerdings zu dieser Zeit auch nicht befremdlich sein müßte, noch über die alternative Ergänzung $\tau \varepsilon \tau \rho \alpha ́ \delta o] \varsigma$ spekulieren.

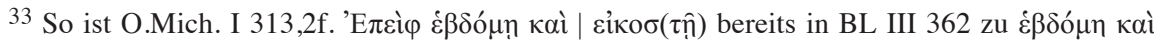

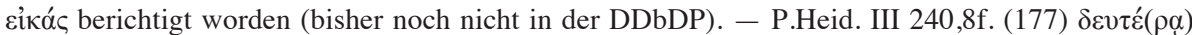

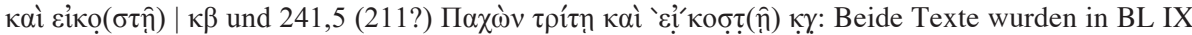

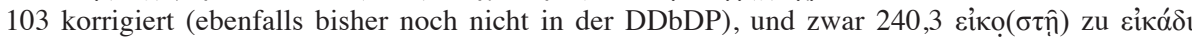

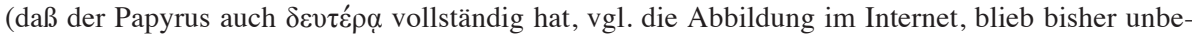

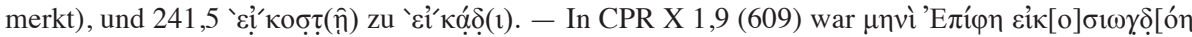

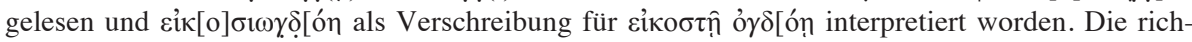

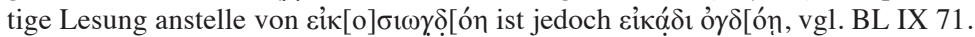




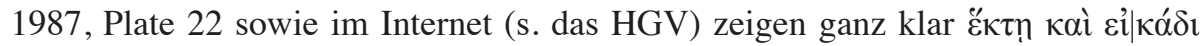
$\kappa \bar{s}$.

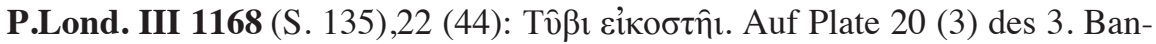

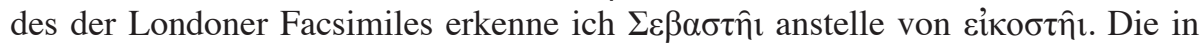
Z. 21f. kopierte Datierung kann also nur auf 28. Dez. 43 - 26. Jan. 44 umgerechnet werden. Daß neben der Bezeichnung $\Sigma \varepsilon \beta \alpha \sigma \tau \eta ́$ das Tagesdatum fehlt, ist unter Augustus und Tiberius die Regel, wird aber unter Claudius äußerst selten; doch s. z.B. O.Bodl. II 484,5 (41-54): П $\alpha \chi \omega)(v) ~ \Sigma \varepsilon \beta \alpha \sigma \tau \hat{n}$. Vgl. W.F. Snyder, Progress Report on the ${ }^{~} H \mu \varepsilon_{\rho} \alpha \imath \Sigma \varepsilon \beta \alpha \sigma \tau \alpha i$, Aegyptus 44 (1964) 145-169 und zuletzt F. Mitthof in Chiron 39 (2009) 200 und N. Gonis in P.Oxy. LXXVIII 5174,10 Komm.

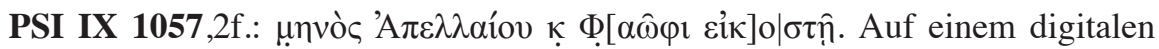
Photo, welches ich J.-L. Fournet verdanke, lese ich 'A $\pi \varepsilon \lambda \lambda \alpha i ́$ ov $\bar{\varepsilon} \Phi[\alpha \hat{\omega}] \varphi[1$ $\left.\pi \varepsilon^{\prime}\right] \mu \mid \ln$. Ein Omikron am Zeilenende erscheint mir unmöglich. Beispiele für die

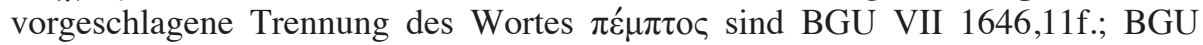
XIII 2349,15f.; O.Ashm. Shelt. 8,3f;; O.Mich. I 357,3f.; O.Mich. I 358,3f.; P.Customs 188,4f.; P.Köln I 52,22f., usw. Das Datum ist dann der 2. Okt. 32.34

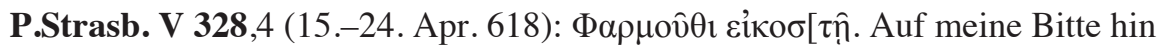
hat P. Heilporn das Original überprüft und mir ein digitales Photo der fraglichen Partie geschickt, wofür ich ihm herzlich danke. Er schreibt am 7. Febr. 2013: „,.. il ne me semble pas impossible de lire sikàc . [ (peut-être un epsilon ensuite, pour

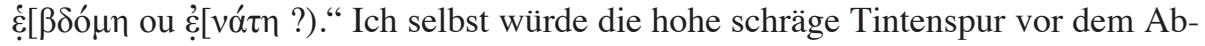
bruch eher dem Schluß-Sigma zurechnen und daher cikàs [ transkribieren.

SB V 7631. Die fragmentarische Doppelurkunde aus dem 20. Regierungsjahr Ptolemaios' III. (228/7 v.Chr.) muß mit den Korrekturen benutzt werden, die W. Clarysse im Jahre 1975 anhand eines Photos gewonnen hat ${ }^{35}$ (= BL VII 195f., aber momentan noch nicht in die DDbDP aufgenommen). Reste der Datierungsformel sind zweifach erhalten, die ich - mit Clarysses Verbesserungen - zur Bequemlichkeit des Lesers beide abdrucke.

Innenschrift:

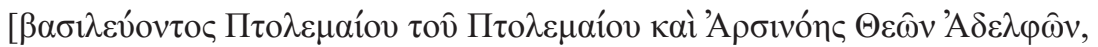

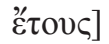

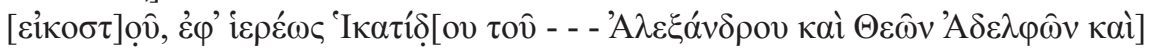

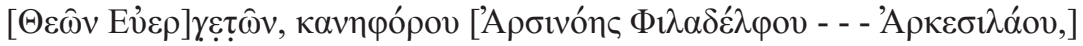

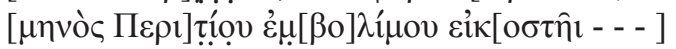

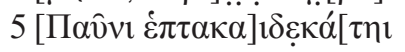

34 Weitere Bemerkungen: Die Korrekturvorschläge P. van Minnens (s. BL VIII 405) sind zutreffend, d.h. in Z. $11 \mathrm{a} \chi \lambda \omega \rho \hat{v} v$ und in Z. $17 \chi \lambda \omega \rho \omega \hat{v}$. Dadurch wird die Änderung in Z. 11 von $\hat{\omega} v$

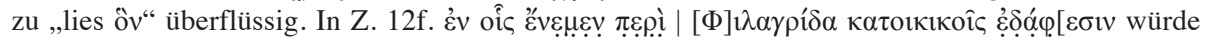

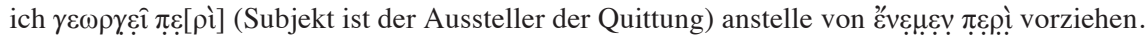

35 W. Clarysse, Notes on Three Papyri Concerning Ptolemaic Clerouchs, AncSoc 6 (1975) 71-77, hier 74-76. 
Außenschrift:

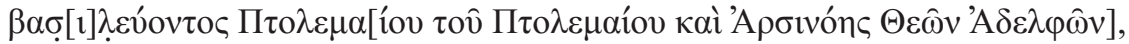
25 ह̌ं

'A $\delta \varepsilon \lambda \varphi \hat{\omega} v]$

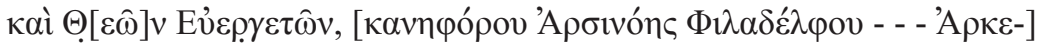

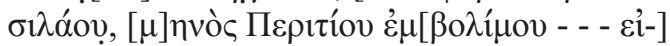

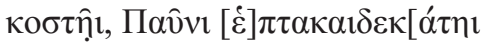

Man wird nach den obigen Ausführungen verstehen, daß in Z. 4 anstelle von

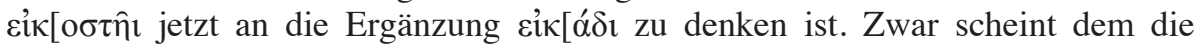

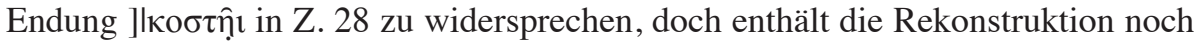

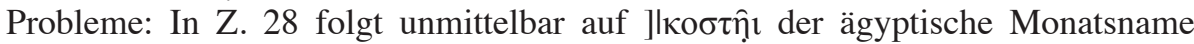
Паv̂vı, was in Z. 4 nicht der Fall ist, sondern es klafft hier eine offene Lücke, die sich in Z. 27 stattdessen nach $\dot{\varepsilon} \mu\left[\beta o \lambda i\right.$ iov auftut. ${ }^{36}$ Willy Clarysse war so freundlich, das Photo, welches er noch besitzt, erneut zu kontrollieren und mir davon auch eine digitale Kopie zu überlassen, wofür ich ihm herzlich danke. Er berichtete per E-Mail am 25. Jan. 2013 zu Z. 28: „... ]kost [ is clear, but the ending is largely lost. [ei] $\mid k o s t[o] u$ would go better." Den Scan vor Augen, kann ich mich diesem Urteil nur anschließen: $\mid \kappa 0 \sigma \tau[0] v$ erscheint mir absolut sicher. Es kann sich also nicht mehr um ein Tagesdatum handeln, sondern nur um eine Jahreszahl, und da wir uns nach Z. 25 in einem 20. Jahr befinden, paßt $\varepsilon i] \mid \kappa o \sigma \tau[o]$ va ganz vorzüglich. Allerdings macht es in einer Doppeldatierung mit Angabe des makedonischen und des ägyptischen Monats keinen Sinn, die Jahreszahl zu wiederholen, es sei denn, die Zählung der Jahre divergierte in den beiden Kalendern. Die Zählung der Jahre muß also in dem Kalender, dem der Payni angehört, dem makedonischen Kalender vorangehen, so

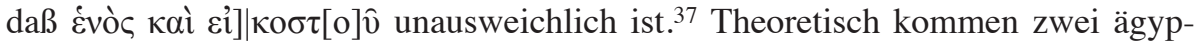
tische Kalender für die Ergänzung in Frage, nämlich das Normal- bzw. Ziviljahr

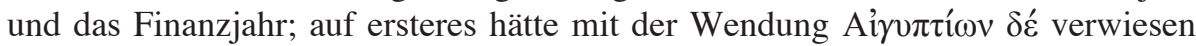
werden können, auf letzteres mit $\dot{\omega} \varsigma \delta$ ai $\pi \rho o ́ \sigma o \delta o 1 .{ }^{38} \mathrm{Da}$ aber das Ziviljahr zu dieser Zeit gewöhnlich später begann als das makedonische Jahr, muß es sich hier um das Finanzjahr handeln, und dieser Schluß wird durch den Umstand gestützt,

${ }^{36}$ Diese Lücke hatte auch schon F. Bilabel, den Herausgeber der Urkunde, irritiert. Er schreibt am Ort der Erstedition (Zur Doppelausfertigung ägyptischer Urkunden, Aegyptus 6 [1925] 93113, hier S. 109, Anm. 1): „Die Kürze von Z. 27 ist mir unverständlich; lag etwa Beschädigung des Papyrus zur Zeit der Beschriftung vor?“. Entsprechend Clarysse, a.a.O., S. 75f.: „There is, however, an obvious irregularity in the dating formula: a good deal of both 1.3 and 1.27 seems to have been left blank, although the lacuna does not occur at exactly the same place (1. 3 after

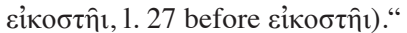

37 Vgl. oben Fußn. 18.

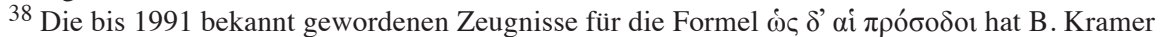
in CPR XVIII, S. 89 zusammengestellt; hinzugefügt werden kann dort P.Enteux. 80,3 (217 v.Chr.):

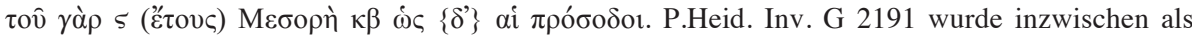
P.Heid. VI 383 ediert, P.Mich. Inv. 6979 als SB XX 15001 abgedruckt. Den sich offenbar verändernden Beginn des Finanzjahres diskutiert R. Duttenhöfer in P.Heid. VI 383,10-12 Komm. 
daß in Z. 13 des fragmentarischen Textes in unklarem Zusammenhang auch von

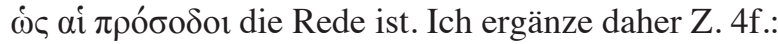

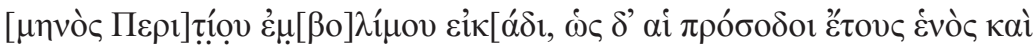

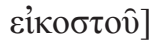

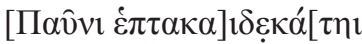

und Z. 27f.:

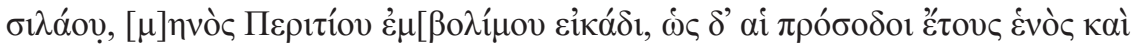
हi-]

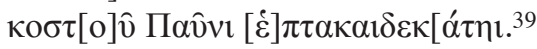

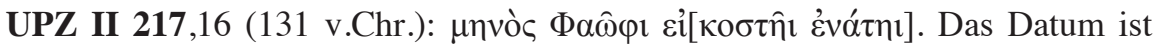

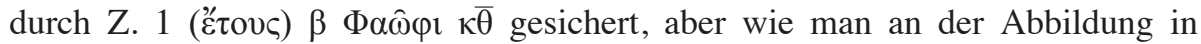
U. Wilcken, Tafeln zur aelteren griechischen Palaeographie, Leipzig u.a. 1891, Tafel IXa (P.Berol. 1383) sieht, ist in Z. 16 vor der Lücke nur das unterste, sich leicht nach links wendende Ende einer Senkrechten erhalten. Statt Iota könnte daher problemlos auch ein Ny rekonstruiert werden, d.h. es wäre auch die zu erwar-

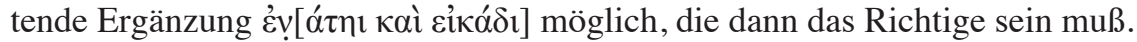

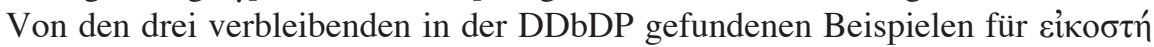
als Bezeichnung eines Monatstages muß eines als augenscheinlich zutreffend angesehen werden, bei einem weiteren bleibt die Entscheidung unbestimmt, und das dritte ist bislang ungeprüft:

In der Edition der Torzollquittung BGU VII 1594,7f. (201 oder 230) steht

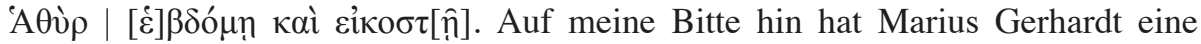
Überprüfung des Originals vorgenommen; sein detaillierter Bericht vom 28. Febr. 2013, für den ich ihm herzlich danke, lautet: „Ich habe mir BGU VII 1594 (= P. 11600) angeschaut und die Lesung der letzten Zeile überprüft. Ich würde eher dem Text der editio princeps zustimmen: Die ersten 3 Buchstaben des letzten Wortes sind sicher zu lesen. Das darauf folgende Omikron ist m.E. ebenfalls sicher zu lesen, zudem haben die Schriftspuren keine Ähnlichkeit mit dem eher ausladenden Alpha an anderen Stellen des Textes. Darauf folgen vor der Fehlstelle im Papyrus noch geringe Schriftspuren, die man als Reste der unteren linken Partie eines Sigma ansprechen könnte. Recht dicht darauf folgt unter der Lücke eine recht große Unterlänge, die zu einem Iota oder Tau gehören $<$ muß>. Der entscheidende obere Teil dieses Buchstabens ist in der Lücke verloren. Danach bricht der Papyrus ab. - Man könnte nun zwar argumentieren, daß es sich bei dem

${ }^{39}$ Durch die neuen Ergänzungen wird die in SB V 7631 dokumentierte Gleichsetzung „20. Peritios embolimos = 17. Payni“ in keiner Weise verändert; der Erkenntnisgewinn liegt darin, daß wir jetzt wissen, daß die Monate verschiedenen Jahreszählungen angehören und deren zweite der ersten vorangeht. $\mathrm{Zu}$ den Implikationen dieser Doppeldatierung und den daraus resultierenden wissenschaftlichen Kontroversen s. zuletzt Chris Bennett, Alexandria and the Moon. An Investigation into the Lunar Macedonian Calendar of Ptolemaic Egypt (Studia Hellenistica 52), Leuven u.a. 2011, S. 66-68 mit Fußn. 6. Das Nebeneinander der drei Kalender (makedonisches Jahr, ägyptisches Ziviljahr, Finanzjahr) veranschaulicht beispielhaft für das 25. Jahr Ptolemaios' III. Bennetts Skizze auf S. 74. 
letzten erhaltenen Buchstaben doch um ein Iota handelt und die Schriftreste davor zu einem Alpha und einem Delta gehören, doch sind beide Buchstaben im Rest des Textes in Aussehen und Größe anders. Gerade für ein Delta wäre auch einfach nicht genügend Platz, so daß ich dem zugegebenermaßen unsicher zu lesenden Sigma den Vorzug geben würde.“

Unsicher bleibt auch der Fall P.Hamb. I 39 (Nr. 30) descr. = P.Rom. Mil.

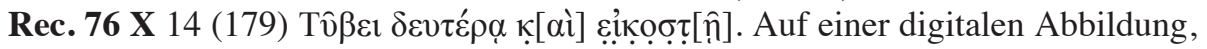
die ich Hans-Walter Stork von der Staats- und Universitätsbibliothek Hamburg verdanke, war ich zunächst geneigt, die Lesung der Edition in der nur leicht modi-

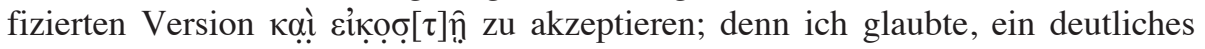
Omikron zu sehen. Doch die Frage, ob denn sikódı wirklich ganz unmöglich sei, muß ich mit „Nein“ beantworten. Entscheidend kommt mir vor, daß das vermeintliche Omikron eine hohe Anbindung nach rechts gehabt haben könnte, was für diesen Buchstaben absolut unpassend wäre. Falls an der Stelle ein Alpha mit dem Aussehen gestanden hätte, welches z.B. auch das erste Alpha von $\delta \eta v \alpha ́ p t \alpha$ in Z. 11 hat, dann wäre die Lesung عikạ́ộ doch vertretbar, wie mein folgender Versuch einer Nachzeichnung verdeutlichen soll. Und wenn દiḳọ́ộ möglich wäre, sollte man ihm vielleicht auch die Präferenz geben.

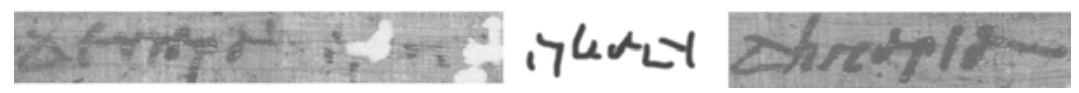

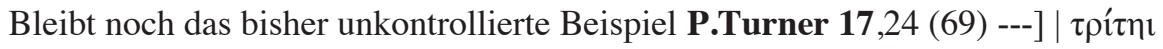

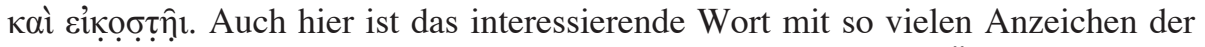
Unsicherheit gedruckt worden, daß die Vermutung, bei einer Überprüfung des Textes könne sich sikád 1 als die korrekte Lesung herausstellen, ohne Zweifel berechtigt ist; die Lesung der Edition bleibt daher suspekt, wenngleich einzuräumen ist, daß ähnlich wie bei BGU VII 1594 und P.Rom. Mil. Rec. 76 X auch in diesem Fall eine Kontrolle möglicherweise keine definitive Klärung brächte. Für das Gesamtergebnis bliebe das unerheblich: Wenn schon zwei potentielle Abweichungen von der Regel vorliegen, würde eine dritte das bisher gewonnene Bild nicht mehr substantiell verändern.

Fazit: Während der gesamten Zeit, in der man Griechisch in Ägypten geschrieben hat, ist દikác sehr konsequent zur Bezeichnung der Tage vom 20. bis zum 29. eines Monats verwendet worden. 385 Belegen für das Zahlsubstantiv stehen nur sehr wenige (d.h. zwei bis drei) mögliche Beispiele für die Verwendung von

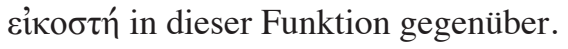

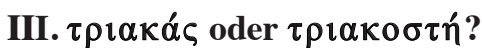

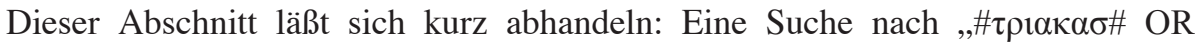
$\# \tau \rho \iota \alpha \kappa \alpha \delta^{\text {“ in papyri.info }}{ }^{40}$ ergab 183 hits, die - von zwei oder drei unsicheren Fällen abgesehen - sich alle auf den 30. Tag eines Monats beziehen. Die frühesten

${ }^{40}$ Durchgeführt am 8. März 2013. 
Zeugnisse stammen aus den Jahren 265 und 256 v.Chr., ${ }^{41}$ und sie reichen bis in

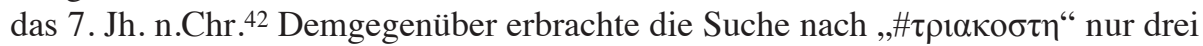
Funde, nämlich die folgenden:

Bei der Edition von P.Tebt. II 483 descr. als P.Tebt. Wall 5 (94) war in Z. 6

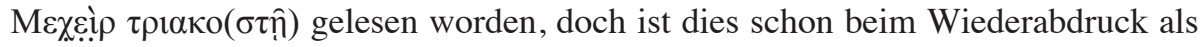

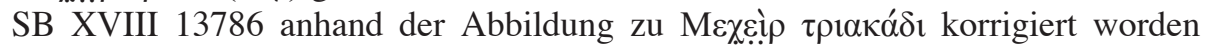
(war zum Zeitpunkt der Abfrage aber noch nicht in die DDbDP übernommen).

In BGU I 240 (Soknopaiu Nesos; ca. 167-169 n.Chr.) liest man in weitgehend

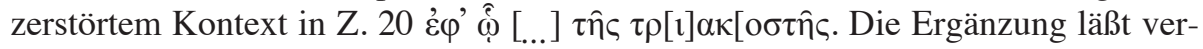
muten, daß die Herausgeber hier an eine Terminsetzung für eine Zahlung „bis zum 30." eines Monats gedacht haben, eine Interpretation, die auch durch das in

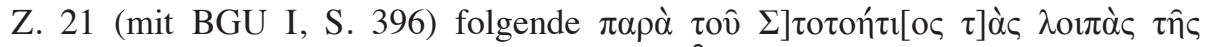

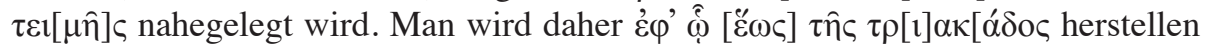
dürfen.

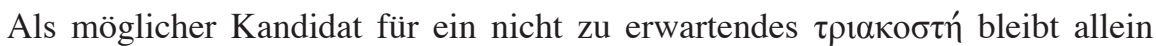

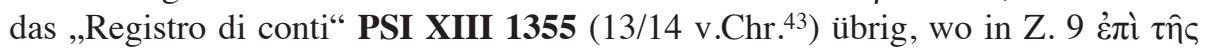

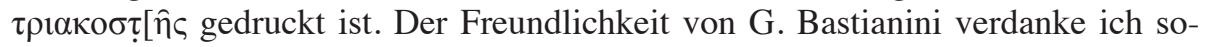
wohl eine digitale Abbildung des Papyrus als auch die Information, daß eine Neuedition des Texts durch Simona Russo in den Comunicazioni XI unter der Nr. 5 unmittelbar bevorsteht, und S. Russo hat mir bereitwillig diese Neuedition noch vor der Veröffentlichung zur Kenntnis gebracht, wofür ich ihr ebenfalls

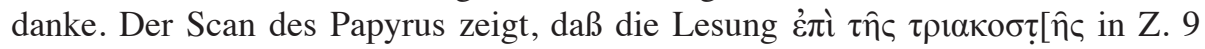
absolut korrekt ist, aber S. Russo betont im Kommentar zu Recht, daß wegen der Lückenhaftigkeit des Texts unklar bleibt, ob mit der Wendung auf den 30. Tag eines Monats verwiesen wird, woran man sicherlich zuerst denken muß, oder ob etwas vollkommen anderes damit bezeichnet wird.

\section{Ergebnis}

In Ägypten wurde ganz offenbar die im Griechischen allgemein geltende Regel, zur Bezeichnung des 4., 20. und 30. Tages eines Monats nicht die Ordinalzahl,

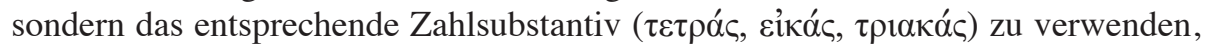
über alle Jahrhunderte hinweg, in denen Griechisch hier die Verkehrssprache war,

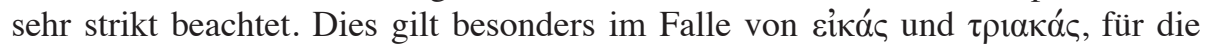
sich hohe Belegzahlen haben finden lassen, während die Gegenbeispiele für

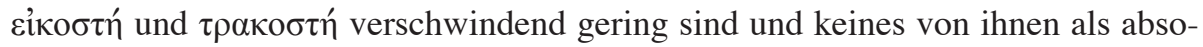

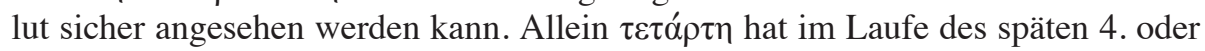

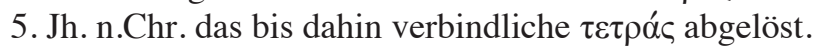

41 P.Strasb. VII 641,4 (265 v.Chr.); P.Cair. Zen. II 59150,8 und PSI V 509,15 (beide 256 v.Chr.).

42 BGU III 725,21 (618); SPP III ${ }^{2}$ 96,5 (640 oder 655).

43 Das ist die Umdatierung (statt 128/7 v.Chr.) durch S. Russo in der oben genannten Neuedition. 